\title{
Green Synthesis, Characterization and Antibacterial Study of Ag-Au Bimetallic Nanocomposite using Tea Powder Extract
}

\author{
Arunkumar Lagashetty ${ }^{1, *(\mathbb{D})}$, Sangappa K Ganiger ${ }^{2}(\mathbb{D})$, Preeti R. K. ${ }^{3(\mathbb{D})}$, Shashidhar Reddy 4 (D) \\ 1 Department of Studies in Chemistry, Vijayanagara Sri Krishnadevaraya University, Ballari, 583105 Karnataka, India \\ 2 Department of Physics, Government Engineering College, Raichur 584135 Karnataka, India \\ sangappaganiger1973@gmail.com (S.K.G.); \\ 3 Department of Zoology, Gulbarga University, Kalaburagi, 585103 Karnataka, India; preetirk999@ gmail.com (P.R.K.); \\ 4 Department of Chemistry, S.D.M College of Engineering and Technology, Dharwad, 580002 Karnataka, India; \\ shashidhar66111@gmail.com (S.R.); \\ * Correspondence: arun.lagashetty@gmail.com;
}

Scopus Author ID 35608190300

Received: 30.06.2020; Revised: 15.07.2020; Accepted: 16.07.2020; Published: 18.07.2020

\begin{abstract}
Synthesis of metal nanoparticles using plant extracts is attracted much to the recent researchers due to its simplicity, which integrates chemical technology. Special attention is given to the green synthesis of nanoparticles by easily available plants with an eco-friendly system compared to other conventional methods. Silver-Gold nanocomposite (Ag-Au NCp's) is synthesized by bi reduction of silver nitrate and gold chloride. These metal salts are simultaneously reduced by tea extract to form respective silver and gold nanocomposite. The structure and morphology of as-prepared Ag-Au NCp's sample were characterized by employing powder X-ray diffraction (XRD) tool and by Scanning Electron Micrograph (SEM) tool, respectively. Fourier Transform Infrared (FTIR) spectral study was undertaken to know the bonding in the prepared silver sample. Morphology and particle size of the above composite was studied by the Transmission electron microscope (TEM) tool. Energy-dispersive X-ray analysis (EDX) study was undertaken to know the formation of Ag-Au NCp's. Antibacterial activity study is undertaken to know its biological behavior.
\end{abstract}

Keywords: Reduction; Ag-Au NCp's; Tea powder extract; EDX; Antibacterial.

(C) 2020 by the authors. This article is an open-access article distributed under the terms and conditions of the Creative Commons Attribution (CC BY) license (https://creativecommons.org/licenses/by/4.0/).

\section{Introduction}

Recent researchers are attracted much towards emerging nanotechnology due to its new properties and applications [1-2]. Future technology will be built by tailoring the blocks of many applications by the use of nanomaterials. Metal nanoparticles have a high specific surface area and a high fraction of surface atoms. Unique physicochemical characteristics of nanoparticles, including catalytic activity, optical properties, electronic properties, antibacterial properties, and magnetic properties, are gaining the importance for their novel methods of synthesis [3-4]. Green preparation of nanomaterials by the use of various plant extraction technique shows enhanced unique properties and applications with proper manipulation compared to its bulk materials [5-7]. Research on the synthesis of metals and metal oxides nanoparticles by biological route, especially by the use of plant extract, is developing in a rapid way because of its simple experimentation and high potential applications. The biological method for the materials synthesis at the nanoscale is in accordance with an eco-friendly system that avoids burning with fuel. Currently, there is a growing need to develop environmentally 
benevolent nanoparticle synthesis processes that do not use toxic chemicals in the synthesis protocol [8-10]. The need for environmental non-toxic synthetic protocols for nanoparticles leads to the developing interest in biological approaches, which are free from the use of toxic chemicals as by-products. Thus, there is an increasing demand for green nanotechnology [1112]. Many biological approaches for both extracellular and intracellular nanoparticle synthesis have been reported using microorganisms, including bacteria, fungi, andplants. The bioreduction method for the synthesis of nanoparticles using plant extracts is the most adopted method for the production of nanoparticles and also has a special advantage that the plants are widely distributed, easily available, much safer to handle, and act as a source of several metabolites [13-14]. Silver and gold nanoparticle prepared by the biological method has attracted considerable interest due to their extensive applicability in various areas such as drug delivery system, electronics, chemical engineering, energy-efficient system and also in the field of medicine [15-17]. The rapidly developing field of nanotechnology will result in the exposure of nanoparticles to humans via several routes (e.g., inhalation, ingestion, skin, etc.). Nanoparticles can translocate from the route of exposure to other vital organs and penetrate cells. Toxicity studies to determine the deleterious effects of nanoparticles on living cells are required. Many techniques of synthesizing silver nanoparticles, such as chemical reduction of silver ions in aqueous solutions with or without stabilizing agents are in literature [18-19]. Sometimes the synthesis of nanoparticles using plants or parts of plants can prove advantageous over other biological processes by eliminating the elaborate processes of maintaining microbial cultures [20-21].

Tea leaves are extensively grown in India and are widely used to prepare a tea powder, which finds much demand by Asian people [22-23]. Production of nanoparticles can be achieved mainly through three methods such as chemical, physical, and biological. Since noble metals such as gold, silver, and platinum nanoparticles are widely applied to human contacting areas, a large variety of possible biomedical applications have been examined [24-25].

The literature survey reviews the different metals and metal oxide nanoparticles are prepared by the biological route successfully, but bimetallic nanocomposite particles by green synthesis route are reported very less. Here is an attempt for the synthesis of Ag-Au NCp's material by reduction of silver nitrate and gold chloride using tea powder. Simultaneous in situ bioreduction method was employed for synthesis Ag-Au NCp's material. The prepared sample is well characterized for its structure by X-ray diffraction (XRD), morphology by Scanning Electron Microscope (SEM), and bonding by Fourier Transform Infrared study (FT-IR) techniques. EDX analysis is carried out for the prepared sample to know the formation of AgAu NCp's. Antibacterial activity study is undertaken to know its biological behavior

\section{Materials and Methods}

\subsection{Materials and Methods.}

The reagents used were of analytical grade obtained from Merck (Mumbai, India). Red lable of Tata, India Ltd, silver nitrate, and gold chloride solution was prepared in distilled water. All glassware are properly rinsed with chromic acid followed by double distilled water and dried. In situ biological reduction of silver nitrate and gold chloride into its nanocomposite by tea powder extract. 


\subsection{Preparation of tea powder extract.}

One gram of red lable tea powder was mixed with $100 \mathrm{ml}$ distilled water and shake well for proper mixing. This solution was boiled thoroughly for ten minutes and is allowed to cool at room temperature. Incubate the solution for half an hour for complete settlement. The reaction mixture was filtered through Whatman filter paper no 40 to get the tea extract.

\subsection{Synthesis of Ag-Au NCp's.}

$1 \mathrm{mM}$ aqueous solutions of silver nitrate and gold chloride are prepared in distilled water in a separate container. These two solutions are equally mixed to form a colorless AgAu salt solution. $10 \mathrm{ml}$ of above-prepared tea extract was added to $100 \mathrm{ml}$ of $1 \mathrm{mM}$ aqueous $\mathrm{Ag}-\mathrm{Au}$ salt solution taken in a conical flask. This reaction mixture was on microwave irradiation, having $2.45 \mathrm{GHz}$ frequency at power $800 \mathrm{w}$ for about 15 minutes. The reaction mixture was incubated for 24 hours at room temperature for complete reduction. Color change from a clear solution to brown (as shown in figure 1) is observed due to the complete reduction of metal salts into its metal nanocomposite. As formed, Ag-Au nanocomposite thus was centrifuged at $500 \mathrm{rpm}$ for about ten minutes, followed by re-dispersion in $10 \mathrm{ml}$ of distilled water. The preparative scheme is represented in figure 2 [26].

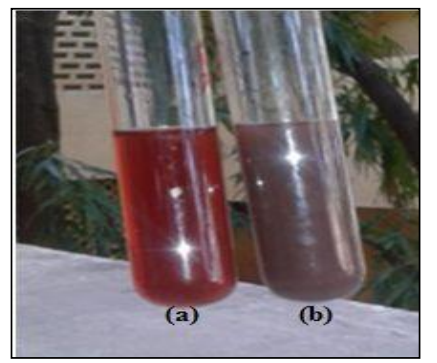

Figure 1. Color of (a) Tea Extract (b) Ag-Au nanocomposite solution.

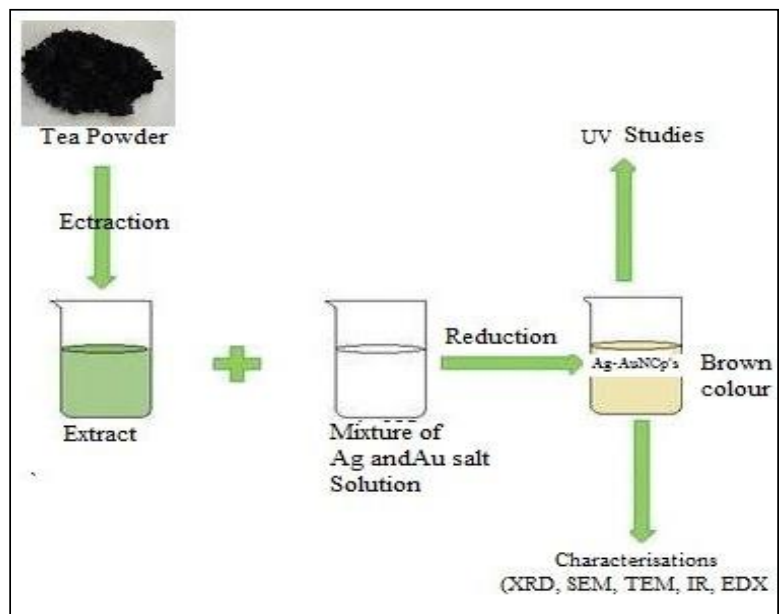

Figure 2. Preparative scheme of Ag-Au Np's using Tea powder extract.

\subsection{Antibacterial activity of biosynthesized Ag-Au NCp's.}

The antibacterial activity of the biosynthesized Ag NPs against Gram-positive and negative bacteria species was done by the disk diffusion method. Experimented bacteria were Bacillus subtilis Bacillus $v$ and K planticola. Bacterial strains were spread on the Petri dishes, which contained autoclaved Luria-Bertani (LB) medium containing agar. Then the disks soaked in distilled water as a control, plant extract, and biosynthesized Ag-Au NCp's were 
separately placed on Petri dishes containing LB media. Petri dishes were incubated at $37{ }^{0} \mathrm{C}$. The inhibition zone of each disk was measured by a ruler after18 $\mathrm{h}$.

\subsection{Characterization.}

The structures of as prepared Ag-Au NCp's were studied by X-ray diffraction using X' Pert Pro X-ray diffractometer with $\mathrm{Cu} \mathrm{K} \alpha$ as a source of radiation in a $\theta-2 \theta$ configuration. Morphology and bonding of the above oxide were studied by Phillips XL 30 FESEM and Perkin-Elmer 1600 spectrophotometer in $\mathrm{KBr}$ medium tools, respectively. The UV visible spectrophotometric measurements were performed on the Elico spectrophotometer. JEOL JSM-6380 LA Scanning electron microscope with energy dispersive microanalysis of X-Ray (EDAX) is used to study particle morphology with metal confirmation of the sample. TEM images are carried out using Technai-20 Philips transmission electron microscope. The transmission electron microscope was operated at $190 \mathrm{KeV}$ [27].

\section{Results and Discussion}

\subsection{X-ray diffraction.}

Figure 3 shows the indexed XRD pattern of as-prepared Ag-Au NCp's sample. The pattern shows the presence of Bragg's reflections due to the crystalline nature of the composite. Some d-spacing values of the sample match well with a standard 87-0720JCPDS file of Ag and some d-spacing values are matched with standard 04-0784 JCPDS file of Au. Unit cell parameters ware identified by the least-square refinement of the XRD data., The presence of both $\mathrm{Ag}$ and $\mathrm{Au}$ reflections in a single XRD pattern indicates the formation of Ag-Au NCp's and is supported by EDX results explained in the later stage.

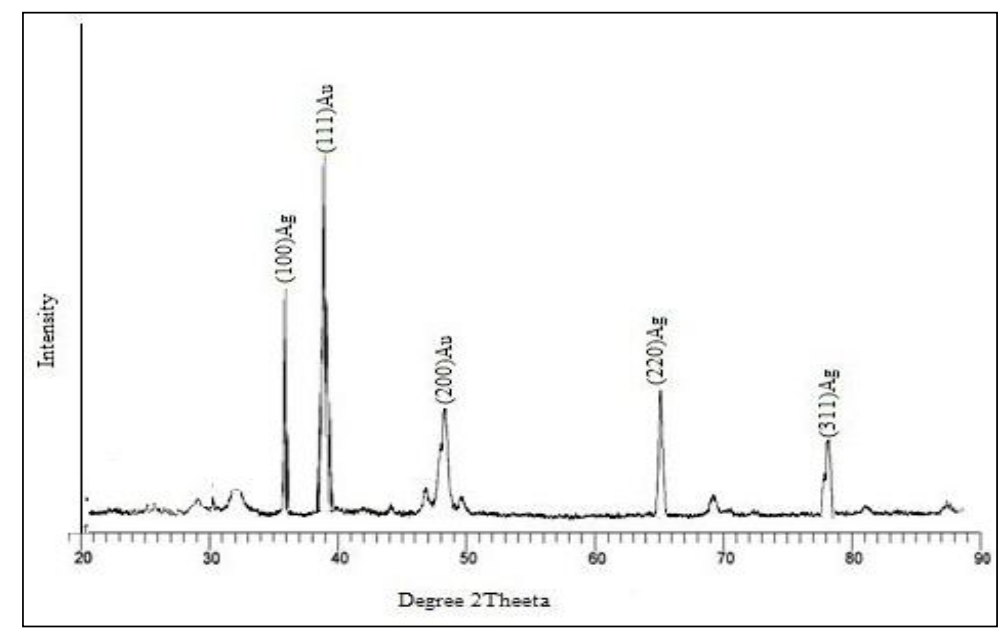

Figure 3. XRD pattern of Ag-Au nanocomposite.

\subsection{Scanning electron microscopy (SEM).}

The morphology of the as-prepared Ag-Au NCp's was studied by scanning electron micrograph tool. Figure 4 shows SEM image of as-prepared Ag-Au NCp's sample. This image shows the crystalline nature with fine spherical particles. Most of the particles are selfassembled with a close compact arrangement. The grain morphology is observed to various properties as well as applications. 


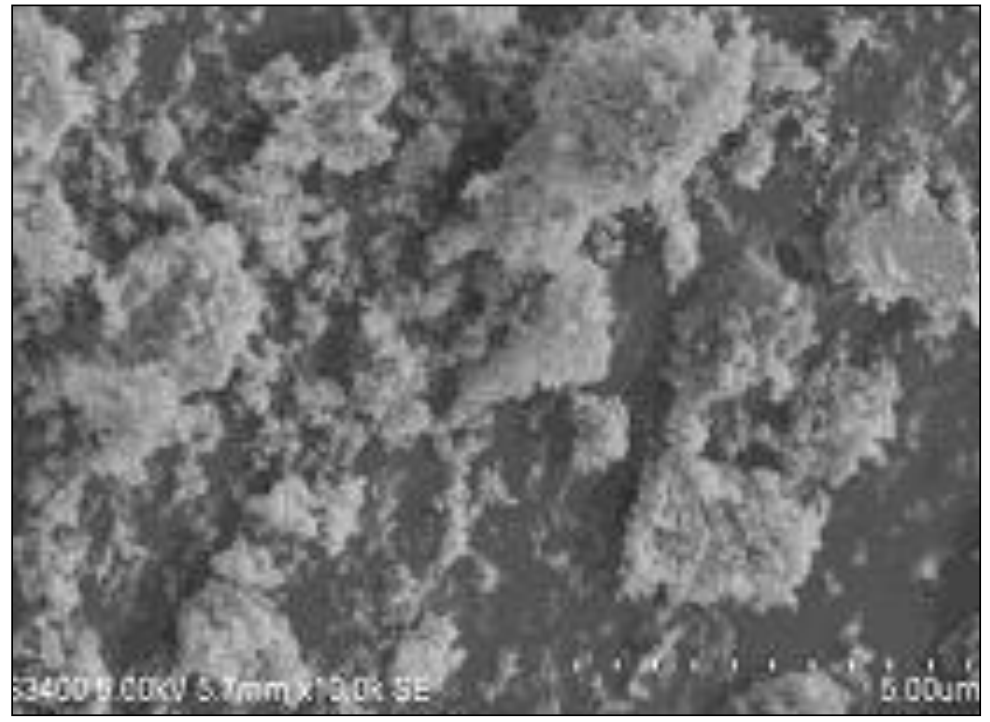

Figure 4. SEM image of Ag-Au nanocomposite.

\subsection{Infrared study.}

The bonding nature of the Ag-Au NCp's was studied by the Infrared tool. The centrifuged and dispersed Ag-Au NCp's obtained has removed free residual biomass. Subsequently, the dried powder was obtained by lyophilizing the purified suspension. The resulting lyophilized powder was examined by the Infrared tool. The obtained frequencies of the sample are given in table 1. The FTIR spectrum of silver nanoparticles showed the band between 3490-3500 $\mathrm{cm}^{-1}$ corresponds to $\mathrm{O}-\mathrm{H}$ stretching H-bonded alcohols and phenols. The peak found around 1500-1550 $\mathrm{cm}^{-1}$ showed a stretch for C-H bond, peak around $1450-1500$ $\mathrm{cm}^{-1}$ showed the bond stretch for N-H. Whereas the stretch for Ag or Au-NPs were found around 500-550 $\mathrm{cm}^{-1}$. Therefore the synthesized nanoparticles were surrounded by proteins and metabolites such as terpenoids having functional groups. From the analysis of FTIR studies, we confirmed that the carbonyl groups from the amino acid residues and proteins have the stronger ability to bind metal, indicating that the proteins involved in the formation metal nanoparticles to prevent agglomeration and thereby stabilize the medium. This suggests that the biological molecules could perform dual functions of the formation and stabilization of silver nanoparticles in the aqueous medium. Carbonyl groups proved that flavanones or terpenoids absorbed on the surface of metal nanoparticles. Flavanones or terpenoids could be adsorbed on the surface of metal nanoparticles, possibly by interaction through carbonyl groups or $\pi$-electrons in the absence of other strong ligating agents in sufficient concentration. The presence of reducing sugars in the solution could be responsible for the reduction of metal ions and the formation of the corresponding metal nanoparticles. It is also possible that the terpenoids play a role in the reduction of metal ions by oxidation of aldehydic groups in the molecules to carboxylic acids [28].

Table 1. Obtained Infrared frequencies of the sample.

\begin{tabular}{l|c} 
Sl. No & Obtained Frequency $\left(\mathrm{cm}^{-1}\right)$ \\
\hline 1 & 3490 \\
\hline 2 & 3500 \\
\hline 3 & 1550 \\
\hline 4 & 1520 \\
\hline 5 & 1450 \\
\hline 6 & 1500 \\
\hline 7 & 550 \\
\hline 8 & 500
\end{tabular}




\subsection{EDX Analysis.}

The presence of silver and gold in the synthesized Ag-Au nanocomposite sample was analyzed by the EDX technique. Figure 5 shows the EDX spectrum of as-synthesized Ag-Au nanocomposite. This pattern shows the presence of both $\mathrm{Ag}$ and $\mathrm{Au}$ atom signals at the respective position. These characteristic absorption peaks of $\mathrm{Ag}$ and $\mathrm{Au}$ confirm the formation of nanocomposite material [29].

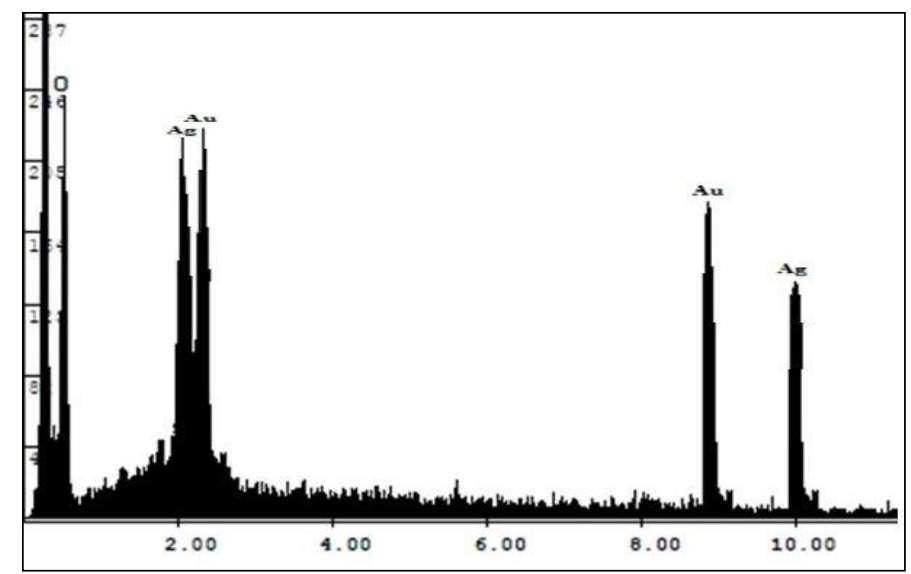

Figure 5. EDX pattern of as-synthesized Ag-Au NCp's.

\subsection{UV-visible study.}

Figure 6 shows UV spectrum of Ag-Au Np's prepared by bioreduction method. The peak at $420 \mathrm{~nm}$ indicates the presence of $\mathrm{Ag}$ nanoparticles. In addition to this, the UV absorption peak at $550 \mathrm{~nm}$ may be assigned due to the gold Au nanoparticle. The shifting towards the red side indicates the formation of a colloidal gel solution containing both Ag and Au.

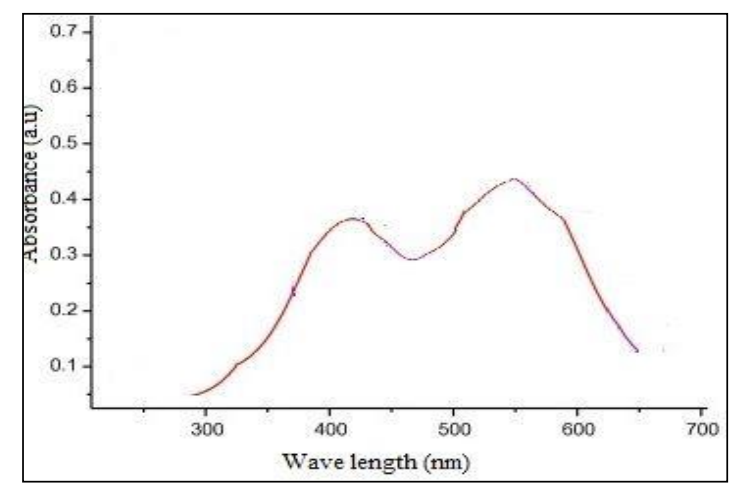

Figure 6. UV Spectrum of Ag-Au NCp's.

\subsection{Transmission electron microscopy (TEM).}

The transmission electron microscope image is scanned to know the perfect morphology and particle size of the prepared Ag-Au NCp's. Figure 7 shows the TEM image of prepared Ag-Au NCp's formed by tea extract solution. The image shows particles are in the nano range, and most of the particles are spherical and also finds varied particle sizes. Bo, the $\mathrm{Ag}$ and $\mathrm{Au}$ particles are well dispersed with each other and form a crystalline nature, which was confirmed by the XRD tool. Close compact structure and fine agglomeration can also be viewed in the image [30]. 


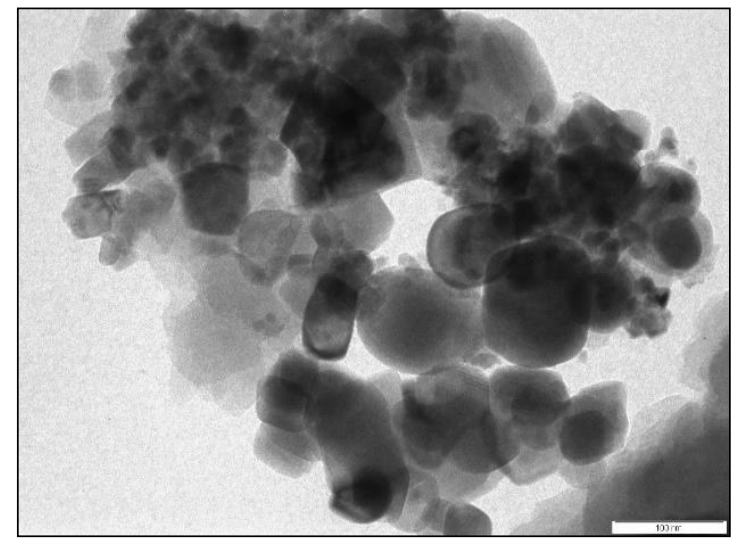

Figure 7. TEM image of Ag-Au NCp's.

\subsection{Antibacterial Activity of Ag-Au NCp's.}

The zone of inhibition measured is summarized in table 2. From the table, it is evident that the synthesized nanoparticles are good candidates for their usage in antibacterial drugs. This sample shows remarkable activity against $S$. subtilis and $K$. planticola bacteria, respectively. The higher activity towards $S$. subtilis compared to $K$. planticola can be viewed. One can find the effect of concentration of the sample for activity towards the higher end [3132].

\begin{tabular}{|c|c|c|c|}
\hline \multirow[t]{2}{*}{ Sl. No } & \multirow[t]{2}{*}{$\begin{array}{l}\text { Concentration of } \\
\text { Ag-Au NCp's }(\mu 1)\end{array}$} & \multicolumn{2}{|c|}{$\begin{array}{c}\text { Bacterial strain } \\
\text { (Zone of inhibition) }\end{array}$} \\
\hline & & S. Subtills & K. Planticola \\
\hline 1 & 10 & 09 & 6 \\
\hline 2 & 20 & 10 & 7 \\
\hline 3 & 30 & 11 & 8.5 \\
\hline 4 & 40 & 12 & 8 \\
\hline 5 & 50 & 12 & 9 \\
\hline
\end{tabular}

\section{Conclusions}

Biosynthesis of Ag-Au NCp's using tea powder extract is a simple, efficient, ecofriendly method and can be used for the synthesis of other metal nanocomposite materials. This method is one of the economic viability methods for the synthesis of nanoparticles. The reduction of silver and gold ions takes place simultaneously. Further detailed characterization, properties, and applications of Ag nanoparticles is the future direction of our work.

\section{Funding}

This research received no external funding.

\section{Acknowledgments}

Authors thank DST-FIST (SR/FST/CSI-003/2016) grant provided for instruments and infrastructure facilities at Vijayanagar Srikrishna Devaraya University Ballari. Thanks are due to Prof. A.Venkataraman, Professor, Department of Chemistry, Gulbarga University, Kalaburagi, Karnataka, India, for useful discussion in spectral analysis. 


\section{Conflicts of Interest}

\section{The authors declare no conflict of interest.}

\section{Refereces}

1. Priya A.,; Saminathan, S.; Balasundaram, J. Green synthesis of NiO nanoparticles using Leucas Asperaand its antibacterial activity, Letters in Applied NanoBioscience 2020, 9(2), 1033-1036, https://doi.org/10.33263/LIANBS92.10331036.

2. Rani, S.; Palle; Penchalaneni, J.; Lavudi, K.; Gaddam, S.A.; Kotakadi, V.S.; Challagundala, V.N. Green Synthesis of Silver Nanoparticles by Leaf Extracts of Boerhavia erecta and Spectral Characterization and Their Antimicrobial, Antioxidant ad Cytotoxic Studies on Ovarian Cancer Cell Lines, Letters in Applied NanoBioscience 2020,9(3), 1165-1176, https://doi.org/10.33263/LIANBS93.11651176

3. Mickymaray, S. One-step Synthesis of Silver Nanoparticles Using Saudi Arabian Desert Seasonal Plant Sisymbrium irio and Antibacterial Activity Against Multidrug-Resistant Bacterial Strains. Biomolecules 2019, 9, 662-669, https://doi.org/10.3390/biom9110662.

4. Ashraf, H.; Anjum, T.; Riaz, S.; Naseem, S. Microwave-Assisted Green Synthesis and Characterization of Silver Nanoparticles Using Melia azedarach for the Management of Fusarium Wilt in Tomato. 2020, 11, 1221, https://doi.org/10.3389/fmicb.2020.00238.

5. Ulaeto, S.B.; Mathew, G.M.; Pancrecious, J.K.; Nair, J.B.; Rajan, T.P.D.; Maiti, K.K.; Pai, B.C. Biogenic Ag Nanoparticles from Neem Extract: Their Structural Evaluation and Antimicrobial Effects against Pseudomonas nitroreducens and Aspergillus unguis (NII 08123). ACS Biomaterials Science \& Engineering 2020, 6, 235-245, https://doi.org/10.1021/acsbiomaterials.9b0125.

6. Hamelian, M.; Varmira, K.; Veisi, H. Green synthesis and characterizations of gold nanoparticles using Thyme and survey cytotoxic effect, antibacterial and antioxidant potential. Journal of Photochemistry and Photobiology B: Biology 2018, 184, 71-79, https://doi.org/10.1016/j.jphotobiol.2018.05.016.

7. Panchal, P.; Paul, D.R.; Sharma, A.; Choudhary, P.; Meena, P.; Nehra, S.P. Biogenic mediated Ag/ZnO nanocomposites for photocatalytic and antibacterial activities towards disinfection of water. Journal of Colloid and Interface Science 2020, 563, 370-380, https://doi.org/10.1016/j.jcis.2019.12.079.

8. Taha, A.; Ben Aissa, M.; Da'na, E. Green Synthesis of an Activated Carbon-Supported Ag and ZnO Nanocomposite for Photocatalytic Degradation and Its Antibacterial Activities. Molecules 2020, 25, https://doi.org/10.3390/molecules25071586.

9. Aritonang, H.F.; Koleangan, H.; Wuntu, A.D. Synthesis of silver nanoparticles using aqueous extract of medicinal plants' (Impatiens balsamina and Lantana camara) fresh land analysis of antimicrobial activity. Int. J.Microbiol 2019,https://doi.org/10.1155/2019/8642303.

10. Saba, G.; Faeze, F.; Saeed, B. Phytochemical Synthesis of Silver Nanoparticles Using Anthemis Nobilis Extract and Its Antibacterial Activity. Zeitschrift für Physikalische Chemie 2020, 234, 531-540, https://doi.org/10.1515/zpch-2018-1288.

11. Killivalavan, G.; Prabakar, A.C.; Naidu, K.C.B.; Rao, B.R. Synthesis and characterization of pure and Cu doped $\mathrm{CeO}_{2}$ nanoparticles: photocatalytic and Antibacterial activities evaluation, Biointerface Research in Applied Chemistry 2020, 10, 5306-5311,

12. Li, S.; Wei, T.; Tang, M.; Chai, F.; Qu, F.; Wang, C. Facile synthesis of bimetallic Ag-Cu nanoparticles for colorimetric detection of mercury ion and catalysis. Sensors and Actuators B: Chemical 2018, 255, 14711481, https://doi.org/10.1016/j.snb.2017.08.159.

13. Thirumagal, N.; Jeyakumari, A.P. Structural, Optical and Antibacterial Properties of Green Synthesized Silver Nanoparticles (AgNPs) Using Justicia adhatoda L. Leaf Extract. Journal of Cluster Science 2020, 31, 487-497, https://doi.org/10.1007/s10876-019-01663-z.

14. Fatima, R.; Priya, M.; Indurthi, L.; Radhakrishnan, V.; Sudhakaran, R. Biosynthesis of silver nanoparticles using red algae Portieria hornemannii and its antibacterial activity against fish pathogens. Microbial Pathogenesis 2020, 138,https://doi.org/10.1016/j.micpath.2019.103780.

15. Tyagi, P.K.; Mishra, R.; Khan, F.; Gupta, D.; Gola, D. Antifungal Effects of Silver Nanoparticles against Various Plant Pathogenic Fungi and its Safety Evaluation on Drosophila melanogaster. Biointerface Research in Applied Chemistry 2020, 10, 6587-6596.

16. Al-Haddad, J.; Alzaabi, F.; Pal, P.; Rambabu, K.; Banat, F. Green synthesis of bimetallic copper-silver nanoparticles and their application in catalytic and antibacterial activities. Clean Technologies and Environmental Policy 2020, 22, 269-277, https://doi.org/10.1007/s10098-019-01765-2.

17. Lagashetty, A.; Patil, M.K.; Ganiger, S.K. Green Synthesis, Characterization, and Thermal Study of Silver Nanoparticles by Achras sapota, Psidium guajava, and Azadirachtaindica Plant Extracts. Plasmonics2019, 14, 1219-1226, https://doi.org/10.1007/s11468-019-00910-3.

18. Fierascu, I.; Fierascu, I.C.; Brazdis, R.I.; Baroi, A.M.; Fistos, T.; Fierascu, R.C. Phytosynthesized Metallic Nanoparticles_-between Nanomedicine and Toxicology. A Brief Review of 2019's Findings. Materials 2020, 13, https://doi.org/10.3390/ma13030574. 
19. Biswas, A.; Vanlalveni, C.; Adhikari, P.P.; Lalfakzuala, R.; Rokhum, L. Green biosynthesis, characterisation and antimicrobial activities of silver nanoparticles using fruit extract of Solanum viarum. IET Nanobiotechnology 2018, 12, 933-938, https://doi.org/10.1049/iet-nbt.2018.0050.

20. Unuofin, J.O.; Oladipo, A.O.; Msagati, T.A.M.; Lebelo, S.L.; Meddows-Taylor, S.; More, G.K. Novel silverplatinum bimetallic nanoalloy synthesized from Vernonia mespilifolia extract: Antioxidant, antimicrobial, and cytotoxic activities. Arabian Journal of Chemistry 2020, 2712, https://doi.org/10.1016/j.arabjc.2020.06.019.

21. Sharma, C.; Ansari, S.; Ansari, M.S.; Satsangee, S.P.; Srivastava, M.M. Single-step green route synthesis of $\mathrm{Au} / \mathrm{Ag}$ bimetallic nanoparticles using clove buds extract: Enhancement in antioxidant bio-efficacy and catalytic activity. Materials Science and Engineering: 116, https://doi.org/10.1016/j.msec.2020.111153.

22. Annu, Akbar Ali,; Shakeel Ahmed. Green Synthesis of Metal, Metal Oxide Nanoparticles, and Their Various Applications. Handbook of Ecomaterials 2019, 2281-2325, https://doi.org/10.1007/978-3-319-68255$6 \_115$.

23. Gulati, S.; Sachdeva, M.; Bhasin, K.K. Various synthetic routes for the preparation of nanoparticles. AIP Conference Proceedings 2018, 1953, 209-215,https://doi.org/10.1063/1.5032550.

24. Rajput, V.; Minkina, T.; Ahmed, B.; Sushkova, S.; Singh, R.; Soldatov, M.; Laratte, B.; Fedorenko, A.; Mandzhieva, S.; Blicharska, E.; Musarrat, J.; Saquib, Q.; Flieger, J.; Gorovtsov, A. Interaction of CopperBased Nanoparticles to Soil, Terrestrial, and Aquatic Systems: Critical Review of the State of the Science and Future Perspectives. Rev Environ Contam \& Toxicol 2020, 252, 5196, https://doi.org/10.1007/398_2019_34.

25. Sharma, D.; Kanchi, S.; Bisetty, K. Biogenic synthesis of nanoparticles: A review. Arabian Journal of Chemistry 2019, 12, 3576-3600, https://doi.org/10.1016/j.arabjc.2015.11.002.

26. Ganiger, S.K.; Murugendrappa, M.V. Lab Scale Study on Humidity Sensing and D.C. Conductivity of Polypyrrole/Strontium Arsenate (Sr3(AsO4)2) Ceramic Composites. Polymer Science, Series B 2018, 60, 395-404,https://doi.org/10.1134/S1560090418030119.

27. Arunkumar, L.; Sangappa, G.K. Microwave-assisted synthesis, characterisation and thermal study of nano sized metal aluminates.Journal of Metallurgy and Materials Science 2018, 60, 139-148.

28. Chand, K.; Cao, D.; Eldin Fouad, D.; Hussain Shah, A.; Qadeer Dayo, A.; Zhu, K.; Nazim Lakhan, M.; Mehdi, G.; Dong, S. Green synthesis, characterization and photocatalytic application of silver nanoparticles synthesized by various plant extracts. Arabian Journal of Chemistry 2020, 12, 1-14, https://doi.org/10.1016/j.arabjc.2020.01.009.

29. Lagashetty, A.; Pattar, A.; Ganiger, S.K. Synthesis, characterization and antibacterial study of Ag doped magnesium ferrite nanocomposite. Heliyon 2019, 5, https://doi.org/10.1016/j.heliyon.2019.e01760.

30. Lagashetty, A.; Ganiger, S.K.; Shashidhar. Synthesis, characterization and antibacterial study of Ag-Au Bimetallic nanocomposite by bioreduction using piper betle leaf extract. Heliyon 2019, 5, https://doi.org/10.1016/j.heliyon.

31. Ganiger, S.K.; Chaluvaraju, B.V.; Ananda, S.R.; Murugendrappa, M.V. A Feasibility Study of Polypyrrole/Zinc Tungstate (Ceramics) Nano Composites for D. C. Conductivity and as a Humidity Sensor. Materials Today: Proceedings 2018, 5, 2803-2810, https://doi.org/10.1016/j.matpr.2018.01.068.

32. Lagashetty, A.; Ganiger, S.K.; Reddy, S. Microwave Derived Nano Sized Zirconium Vanadate as an Adsorbent for Heavy Metal Ions, Letters in Applied NanoBioscience 2020, 9(3), 1420-1426, https://doi.org/10.33263/LIANBS93.14201426. 